\title{
Vibro-acoustic Response Analysis and Experimental Verification of Aircraft Based on Statistical Energy Analysis
}

\author{
Zhang Guojun \\ School of Mechanics, Civil and Architecture \\ Northwestern Polytechnical University \\ Xi'an ,China \\ zgj2004@163.com
}

\author{
Yan Yunju \\ School of Mechanics, Civil and Architecture \\ Northwestern Polytechnical University \\ Xi'an ,China \\ yjyan_2895@nwpu.edu.cn
}

\begin{abstract}
The SEA (Statistical Energy Analysis) model of the hypersonic aircraft is established based on the SEA theory. Three parameters of the SEA model are determined by the theory, experiential formula and experimental data. Compared the simulation with the experiment, the results of numerical simulation and experiments are basically equal in high frequency domain, but there are much differences in low-frequency. The response sound pressure in aircraft cabin between simulation and experiment is consistent with each other well in both low and high frequency. The errors between them are less than $3 \mathrm{~dB}$. It shows that the method of statistical energy analysis is reliable in the prediction of vibration and noise environment.
\end{abstract}

Keywords: aircraft, statistical energy analysis, parameters determination, vibro-acoustic response, experimental verification.

\section{INTRODUCTION}

Aircraft flying at high-speed will endure the complex and inclement mechanical condition. In the early days of its development, the prefigure of mechanical condition is one of the pivotal links that people always look forward to solving in the process of the design and experiment, and also the important basis of the aircraft design and validated experiment.

Traditional modal analysis based on finite element has a long history in application to dynamic problem on engineering structure system, but this kind of method can be only used to analyze the low-order modal which can be identified clearly. The statistical energy analysis method is suitable to the coupled dynamic problem of the complex system with high frequency and high modal density, including acoustic and structure, structure and structure[1].

In this paper, the structure model of $\mathrm{X}-43 \mathrm{~A}$ is established based on statistical energy analysis theory. In order to study the property and regular pattern of vibration caused by aerodynamic noise, the external noise field is assumed known. The noise transmission and structure coupling vibration are analyzed by SEA method. The structural vibration response and the noise field response of aircraft model are obtained. The reliability of the theory and numerical calculation is verified by noise experiment.

\section{SEA MODELING OF THE AIRCRAFT STRUCTURAL MODEL.}

The aircraft main structure is constructed using $3 \mathrm{~mm}$ uniform thickness plate, and rectangle window is opened below the middle of the cabin to connect the removable engine chassis. The structural material is the stainless steel plate (304), its mechanical physical parameters are, the density $\rho 7800$ $\mathrm{kg} / \mathrm{m}^{3}$, the Elastic modulus E $2.1 \mathrm{e}+11 \mathrm{~Pa}$, the shear modulus $\mathrm{G}$ $8 \mathrm{e}+10 \mathrm{~Pa}$, and Poisson's ratio $\mu$ 0. 3125 .

Generally, there are two problems needed to be solved in building the SEA model. One is how to determine the main modal group of the structural vibration, and the other is the connection among the sub-structures, which will determine the scale of the vibration energy [2]. The established SEA model of X-43A is, shown in Fig. 1. It is simplified as a shell structure aircraft with hollow interior and is looked as the plate-shell structure. The energy transfer among the various subsystems is shown in Fig. 2.

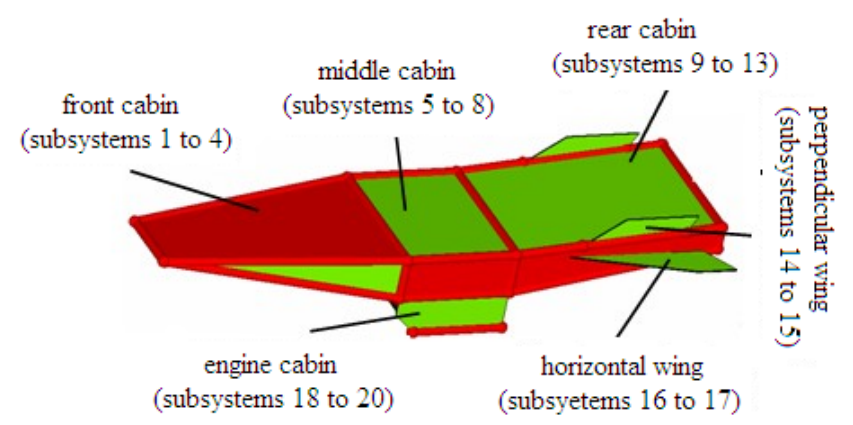

Fig. 1 SEA model of the hypersonic aircraft

According to the principle of dividing sub-structure, combined with the natural geometry, material and energy storage method, the aircraft model is divided into 20 sub-structures including the front cabin, middle cabin, rear cabin, engine cabin, perpendicular wing, horizontal wing et al, and an acoustic cavity subsystem. 


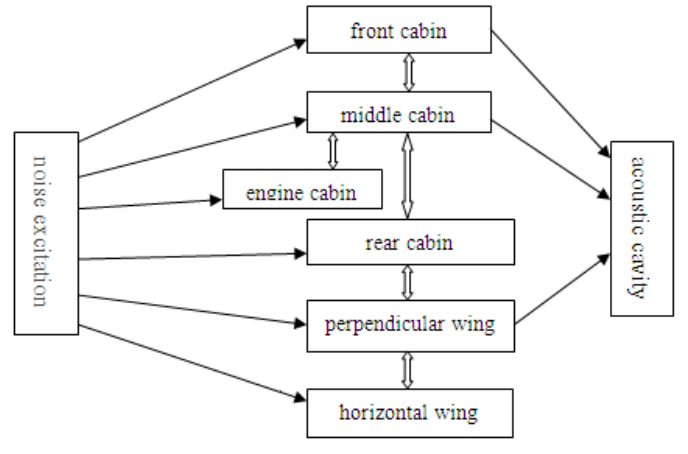

Fig. 2 Transmission path of energy flow

\section{SEA THEORY AND MODELING}

The modal density $n$ (f) in statistical energy analysis is defined as the modal numbers in unit frequency $(\mathrm{Hz})$, it is a physical quantity to describe the stored energy capacity of a vibration system. The estimation formula of modal density of plate was given in references [4] and [5] as follow:

$$
n(f)=n(\omega)=\frac{s \sqrt{3}}{c t} \text {. }
$$

where $S$ is the plate area, $t$ is the plate thickness, and $c=\sqrt{E / \rho\left(1-\mu^{2}\right)}$ is the longitudinal wave velocity.

The estimation formula of the modal density for three-dimensional was given by [6]:

$$
n(f)=n(\omega)=\frac{4 \pi f^{2} V_{0}}{C_{a}{ }^{3}}+\frac{\pi f A}{2 C_{a}{ }^{2}}+\frac{L}{8 C_{a}} .
$$

where $V_{0}$ is the volume of the acoustic field, $\mathrm{A}$ is the acoustic cavity surface area, $L$ is the sideline total length of acoustic cavity, and $C_{a}$ is the acoustic velocity.

Another important parameter in SEA is damping loss factor $\eta_{i}$, and it is composed of the three mutually independent components, i.e.:

$$
\eta_{i}=\eta_{i s}+\eta_{i r}+\eta_{i b}
$$

where $\eta_{i s}$ is the structural loss factor produced by internal friction, $\eta_{\text {ir }}$ is the loss factor produced by vibration sound radiation, and $\eta_{i b}$ is the loss factor produced by boundary connection damping of subsystem. In this study, the connections among the subsystem is rigid, i.e., $\eta_{i b}=0$, so damping loss factor $\eta_{i}$ can be written as $\eta_{i}=\eta_{i s}+\eta_{i r}$. The $\eta_{i s}$ is determined by the material of the $i^{\text {th }}$ subsystem. According to reference [9], we can get the loss factor of stainless steel structure $\eta_{\text {is }}$ is $5 \times 10^{-4}$. The loss factor $\eta_{i r}$ is given by as follows:

$$
\eta_{\text {ir }}=\rho_{0} c \sigma_{i r} / \omega \rho_{s} .
$$

where $\rho_{s}$ and $\sigma_{i r}$ are the area mass density and radiation ratio of subsystems, respectively.

Based on the reverberation time of the acoustic field [6], the damping loss factor can be written as:

$$
\eta_{i}=\frac{2.2}{f T_{R}}=\frac{13.82}{\omega T_{R}}, \quad T_{R}=\frac{55.2 \mathrm{~V}}{c \alpha S} .
$$

Thus,

$$
\eta_{i}=0.04 \mathrm{c} \alpha \mathrm{S} / \mathrm{fV}
$$

where $S$ and $V$ are the area and volume of the acoustic field, $C$ is the velocity of the acoustic field and $\alpha$ is the absorption coefficient

For the SEA model of the aircraft, the coupling loss factors $\eta_{i j}$ includes the coupling between the sub-structures and the coupling between shell and acoustic cavity.

Coupling loss factor of the line connection between two sub-structures is given by [7]:

$$
\eta_{i j}=\frac{2 c_{B i} L \tau_{i j}}{\pi \omega S_{i}}
$$

where $C_{B i}$ is the bending wave velocity of the $i^{\text {th }}$ plate, $L$ is the length, $\omega$ is the center frequency of the band, $S_{i}$ is the area of the $i^{\text {th }}$ subsystem, $\tau_{i j}$ is the wave propagation coefficient of the straight line connecting subsystem between $i$ and $j$.

Coupling loss factor between structure and acoustic field is given by [7]:

$$
\eta_{s v}=\frac{\rho_{0} c \sigma}{\omega \rho_{s}} .
$$

where subscript $\mathrm{s}$ and $\mathrm{v}$ represent the structure and acoustic cavity, $\rho_{0}$ and $\rho_{s}$ are the density, $\sigma$ is the sound radiation coefficient, and $\mathrm{c}$ is the sound velocity.

The input power from external excitation has different calculation method for different practical problems. In this study, the input power is determined by the experiment.

The SEA model of the aircraft consists of 21 sub-systems. In the case of the conservative and weak coupling, when the system undergoes non-related steady-state excitation, based on the motion equations of the system with the modal method, the fluctuation method or the Green's function, the power flow balance equation of subsystem [6] can be written as:

$$
\begin{array}{r}
P_{i i n}=P_{i d}+\sum_{j=1, j \neq i}^{21}\left(\omega \eta_{i j} E_{i}-\omega \eta_{j i}\right)= \\
=\sum_{k=1}^{21} \eta_{i k} E_{i}-\omega \sum_{\substack{j=1 \\
j \neq i}}^{21} \eta_{j i} E_{j}, \\
(l, \varphi=1,2,3, \square, 21) .
\end{array}
$$

where $P_{i i n}$ is the input power of the $i^{\text {th }}$ subsystem, $P_{i d}$ is the loss power of the $i^{\text {th }}$ subsystem. 
The Eq. (9) can be written as:

$$
\begin{aligned}
& \sum_{j=1}^{21} L_{i j} E_{j}=\frac{P_{i i n}}{\omega}(l, \varphi=1,2,3, \square, 21) . \\
\text { where } L_{i j}= & \left\{\begin{array}{ll}
-\eta_{j i} & i \neq j \\
\sum_{K=1}^{21} \eta_{i k} & i=j
\end{array} .\right.
\end{aligned}
$$

Substituting the reciprocity principle relationship $n_{i}(\omega) \eta_{i j}=n_{j}(\omega) \eta_{j i}$ to Eq.(6), one can obtain the statistical energy analysis relation as follows:

$$
\begin{gathered}
{[A]=\left[\begin{array}{cccc}
\left(\eta_{1}+\sum_{i \neq 1}^{k} \eta_{1 i}\right) n_{1} & -\eta_{12} & \ldots & -\eta_{1 N} n_{1} \\
-\eta_{21} n_{2} & \left(\eta_{1}+\sum_{i \neq 2}^{k} \eta_{2 i}\right) n_{2} & \ldots & -\eta_{2 N} n_{2} \\
\ldots & \ldots & \ldots & \ldots \\
-\eta_{N 1} n_{N} & \ldots & \ldots & \left(\eta_{1}+\sum_{i \neq N}^{k} \eta_{N i}\right) n_{N}
\end{array}\right],} \\
\omega[A]=\left[\begin{array}{c}
E_{1} / n_{1} \\
E_{2} / n_{2} \\
\ldots \\
E_{21} / n_{21}
\end{array}\right]=\left[\begin{array}{c}
P_{1} \\
P_{2} \\
\ldots \\
P_{21}
\end{array}\right] .
\end{gathered}
$$

Where $n_{i}(\omega)$ is the modal density of the $i^{\text {th }}$ subsystem and $\mathrm{N}$ is 21 .

Thus, the vibration energy of each subsystem can be solved from Eq.(11).

\section{ANALYSES OF CALCULATION AND EXPERIMENT RESULTS}

In the experiment of structural vibration excited by noise, the mechanical boundary conditions of the structural model is set as free and the external noise excitation field is reverberation noise with frequency range of $20-8000 \mathrm{~Hz}$ and the noise intensity $140-160 \mathrm{~dB}$. Broadband noise vibration experiment was completed in high acoustic intensity reverberation chamber, the experiment site and hoisting way are as shown in Fig. 3.

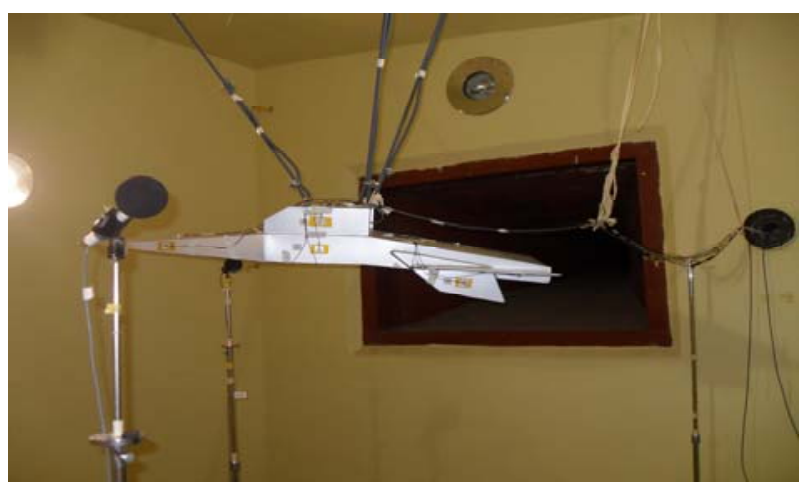

Fig.3 Experiment of structural vibration excited by noise
A. Contrast between the experimental and calculated results of the noise response.

Under the different exciting noise strength, the response noise pressures of the structural cabin are calculated experimented, respectively. Comparison of the experimental and calculated results of the noise response in the structural cabin under the $160 \mathrm{~dB}$ exciting noise is shown in Fig.4.

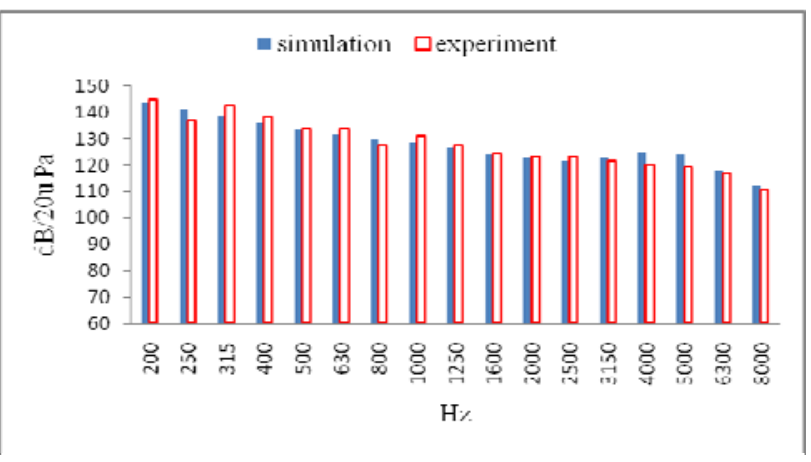

Fig.4 Comparison of the experimental and calculated resultsof the noise response in the structural cabin (exciting noise 160dB)

One can find from Fig.4, the pressure levels of the calculated and experimental response sound in the cabin are rather consistent from low-frequency to high-frequency, and the average error is less than $3 \mathrm{~dB}$. This indicates that the statistical energy analysis is reliable to noise response prediction. Results also show that, the calculated value is less than the experiment in the low frequency band while greater in the high frequencyrange. The smallest error is in the middle frequency band. The frequency of the response sound pressure is widely distributed within the range of $200-8000 \mathrm{~Hz}$, and the amplitude only attenuates about $20 \mathrm{~dB}$ from low to high frequency.

\section{B. Contrast and analysis between the experimental and} calculated results of the structural vibration response.

The structural vibration responses under the different exciting noise strength also are numerically calculated and experimental measured, respectively. Comparison of the experimental and calculated results of the structural vibration response of a given substructure under the $150 \mathrm{~dB}$ and $160 \mathrm{~dB}$ exciting noise is shown in Fig.5. The results show that, the calculated values of the noise-induced structural vibration based on statistical energy analysis are basically consistent with the experiment results in whole frequency range, and is more consistent in the high frequency. The PSD amplitude of each subsystem decrease with the frequency increase. There is a clear peak at high frequency domain $(4000-5000 \mathrm{~Hz})$, which is the important characteristic of broadband noise-induced vibration and shows that the structural vibration response in the high-frequency can not be ignored. 


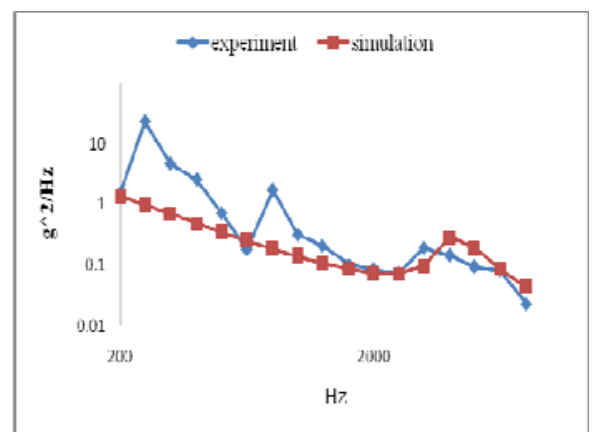

(a) Exciting noise $150 \mathrm{~dB}$

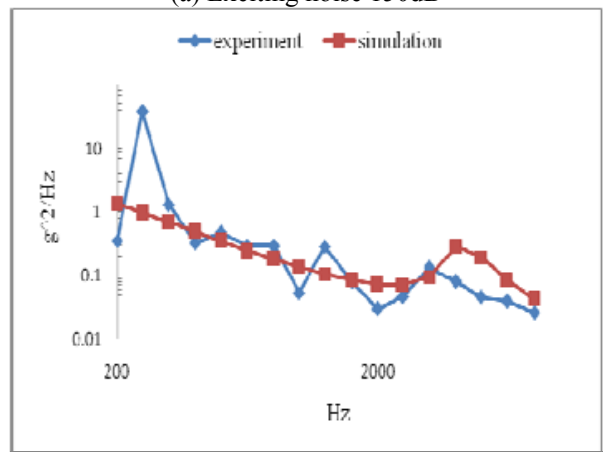

(b) Exciting noise $160 \mathrm{~dB}$

Fig.5 Comparison of the experimental and calculated results of the structural vibration response

\section{SUMMARY}

Taken the $\mathrm{X}-43 \mathrm{~A}$ as a research model, the structure is divided to 21 subsystems according to the basic idea of SEA.
Assumed that the coupling between subsystems is conservative, the SEA model was established, and the required parameters were determined through theory, empirical formula or experimental data. Compared the numerical simulation results with experimented results, the results of the noise response and structural vibration response are well accord to the experimental results. This indicates that the statistical energy analysis method is a credible research method to solve the dynamics problems in the high frequency at present. It can be applied successfully to the analysis of mixed acoustic field, and lay the foundation for further accurate indication of the noise and vibration environment about the aircraft instruments and equipments.

\section{REFERENCES}

[1] Liu Haisheng, Yang Chunzhuang, Chen Shiji: Statistical Energy Analysis Methods Sound Vi- bration Prediction Applied Research[J]. Acoustic technology, 2010,29(2): 192-197.

[2] Wang Xiancheng, Zhang Jing, more clouds. Calculated based on the boats cabin noise modeling statistical energy analysis [J]. Ordnance Technology, 2007,28 (11): 1367-1372.

[3] Laurie A.Marshall Griffin P.Corpening A Chief En-gineer's View of the NASA X-43A Scramjet FlightTest [C]. AIAA/CIRA 13th International Space Planes and Hypersonics Systems and Technol ogies, AIAA, 2005-3332.

[4] Cremer L. Heckl M. and Ungar E.E.,structure-borne sound ,Springer-Verlag, 1973.

[5] Ver I.L.and Holmer C.I. ,Interaction of sound waves with solid structures ,chapter 11 in Noise and Vibration Control,1971.

[6] Yaode Yuan, Wang Qizheng. Statistical energy analysis theory and its application [M] Beijing: Beijing Institute of Technology Press, 1995.

[7] Relevancy, Zhu Shijian, A Summary of Statistical Energy Analysis Method and Its Loss Factor Review on determination [J]. Ship engineering,2004, 26 (4) :10-15. 